Jefferson Wladimir Tenório de

Oliveira'

Ohttps://orcid.org/0000-0002-9656-2256

Ana Paula Nogueira de Magalhães² OChtps://orcid.org/0000-0002-5071-0778

Alice Correia Barros ${ }^{3}$

Ohttps://orcid. org/0000-0002-2627-7185

Elaine Kristhine Rocha Monteiro ${ }^{4}$ Ohttps://oridi.org/0000-0001-6122-3281

Carlos Dornels Freire de Souza

(D) http://orcid.org/0000-0003-0837-8254

Verônica de Medeiros Alves ${ }^{6}$ (-) https://orcid.org/0000-0002-4343-2941

\section{Características das tentativas de suicídio atendidas pelo serviço de emergência pré-hospitalar: um estudo epidemiológico de corte transversal}

\author{
The characteristics of suicide attempts assisted by first \\ responders: a cross-sectional epidemiological study
}

DOI: $10.1590 / 0047-2085000000289$

\section{RESUMO}

Objetivo: Descrever o perfil das vítimas de tentativas de suicídio atendidas pelo Corpo de Bombeiros de um município do semiárido brasileiro. Métodos: Estudo transversal contendo dados do atendimento a vítimas de tentativas de suicídio, realizado pelos bombeiros militares do estado de Alagoas, no período de $1^{\circ}$ de janeiro de 2000 a 31 de dezembro de 2017. Foram selecionadas as variáveis: sexo, idade, desfecho, intoxicação, uso de álcool, transtorno mental, estação do ano, dia da semana, horário, natureza da ocorrência e tempo de resposta, de transporte e total. Foram utilizados os testes qui-quadrado, exato de Fisher e Kruskal-Wallis. Resultados: Foram atendidas 144 vítimas de tentativas de suicídio. A frequência de atendimentos entre homens $(n=73 ; 50,7 \%)$ e mulheres $(n=71 ; 49,3 \%)$ foi semelhante. As mulheres apresentaram idade mais jovem $(p<0,001)$ e utilizaram o método da intoxicação $(p<0,001)$ duas vezes mais que os homens. A presença de transtorno mental $(p=0,04)$ foi mais comum entre os homens e o tempo consumido durante o atendimento pré-hospitalar foi maior entre eles, indicando que, possivelmente, as tentativas de suicídio cometidas por homens demandam maiores cuidados durante a assistência realizada pelos bombeiros. Conclusões: $O$ perfil das vítimas de tentativas de suicídio apresentou frequência semelhante entre homens e mulheres, sendo as mulheres mais jovens. Nas muIheres, observou-se predomínio de intoxicações. Em homens, os transtornos mentais foram mais prevalentes. Além disso, fazem-se necessários o reconhecimento precoce dos casos de tentativas de suicídio, as principais formas de atuação e o preenchimento adequado das fichas de atendimento pré-hospitalar.

\section{PALAVRAS-CHAVE}

Tentativa de suicídio, suicídio, atendimento pré-hospitalar, bombeiros, epidemiologia.

\begin{abstract}
Objective: To describe the profile of suicide attempts assisted by the Fire Department of a Brazilian semi-arid municipality. Methods: This is a cross-sectional study, containing data of care provided to victims of suicide attempts, conducted by the military firefighters of the state of Alagoas, from January 1, 2000 to December 31, 2017. The following variables were selected: sex, age, outcome, intoxication, alcohol use, mental disorder, season of the year, day of the week, schedule, nature of occurrence, response time, transportation time and total time. The chi-square, Fisher's exact test and Kruskal-Wallis tests were used. Results: Between 2000 and 2017, 144 victims of suicide attempts were cared. The frequency of care between men $(n=73 ; 50,7 \%)$ and women $(n=71 ; 49,3 \%)$ was similar. The women showed younger age $(p<0,001)$ and used the intoxication method $(p<0,001)$ twice as often as the men. The presence of mental disorder $(p=0,04)$ was more common among the men and the time consumed during prehospital care was higher among them, indicating that, possibly, suicide attempts committed by men require greater care for the assistance provided by firefighters. Conclusions: The profile of victims of suicide attempts showed a similar frequency between men and women, with younger women. In women, there was a predominance of intoxications. Besides that, mental disorders were more prevalent. However, it is necessary to early recognition of cases of suicide attempts, the main forms of action and the adequate completion of pre-hospital care forms.
\end{abstract}

KEYWORDS

Suicide attempted, suicide, prehospital care, firefighters, epidemiology.

Received in: July/1/2019 Approved in: Jun/22/2020

1 Corpo de Bombeiros Militar de Alagoas, Maceió, AL, Brasil; Universidade Federal de Alagoas (UFAL) - Campus Arapiraca, AL, Brasil.

2 Universidade de São Paulo (USP), Escola de Enfermagem, São Paulo, SP, Brasil; Universidade Federal de Alagoas (UFAL) - Campus Arapiraca, AL, Brasil.

3 Universidade Estadual de Ciências da Saúde de Alagoas (Uncisal), Maceió, AL, Brasil; Universidade Federal de Alagoas (UFAL), Maceió, AL, Brasil.

4 Corpo de Bombeiros Militar de Alagoas, Maceió, AL, Brasil; Universidade Federal de Alagoas (UFAL), Maceió, AL, Brasil; Fundação de Ensino Superior de Olinda (Funeso), Olinda, PE, Brasil.

5 Universidade Federal de Alagoas (UFAL) - Campus Arapiraca, AL, Brasil.

6 Universidade Federal do Rio de Janeiro (UFRJ), Instituto de Psiquiatria, Rio de Janeiro, RJ, Brasil; Universidade Federal de Alagoas (UFAL), Maceió, AL, Brasil. Address for correspondence: Jefferson Wladimir Tenório de Oliveira, Rua Frei Damião de Bozano 44, Boa Vista - 57303-240 - Arapiraca, AL, Brasil. E-mail: jeffersonwto@hotmail.com 


\section{INTRODUÇÃO}

O suicídio é um fenômeno complexo, reconhecido como um grave problema de saúde pública em todo o mundo. Segundo dados da Organização Mundial de Saúde' (OMS), cerca de 800 mil pessoas morrem anualmente por suicídio, tornando-o, atualmente, a segunda maior causa de morte entre jovens de 15 a 29 anos. Quase 80\% dessas mortes concentram-se nos países de baixa e média rendas. Ademais, sabe-se que, para cada adulto que comete o suicídio, há pelo menos vinte outros que tentarão cometê-lo!

No Brasil, o crescimento das taxas de suicídio aumentou entre 200\% e 400\% nas últimas duas décadas e, independentemente da delimitação precisa desse índice, sabe-se que o risco de morrer por suicídio no país está aumentando consideravelmente ${ }^{2}$. Dados do Ministério da Saúde ${ }^{3}$ mostram que, no período de 2011 a 2017, foram registrados 80.352 óbitos por suicídio, dos quais 21.790 (27,3\%) ocorreram na faixa etária de 15 a 29 anos, apontando para um aumento de 10\% na taxa de mortalidade para esse grupo etário. Além disso, nesse mesmo período, um quarto $(25,3 \%)$ das pessoas que cometeram suicídio residia na região nordeste do país ${ }^{3}$.

Os comportamentos suicidas não fatais podem ser classificados em três categorias ${ }^{4}$ : a ideação suicida, que se refere a pensamentos que expressam a vontade de acabar com a vida; o plano suicida, que é a formulação de um método específico pelo qual se pretende morrer; e a tentativa de suicídio, que se refere ao envolvimento em comportamento potencialmente prejudicial no qual existe a intenção de morrer. Apesar de não ser considerado uma doença, o comportamento suicida está associado a diversos transtornos mentais, dos quais os mais frequentes são os transtornos do humor, os transtornos por uso de substâncias, a esquizofrenia e os transtornos de personalidade 5 . Além dos transtornos mentais, o comportamento suicida está relacionado a indicadores sociodemográficos e clínicos, tais como: antecedentes familiares; sexo e idade; estar desempregado ou aposentado; ausência de um companheiro; e histórico de abuso na infância ${ }^{5,6}$.

Diante da magnitude desse fenômeno, a OMS publicou uma série de manuais destinados a grupos específicos que podem atuar consideravelmente na prevenção do suicídio. Entre essas publicações, encontra-se um documento destinado a policiais, bombeiros e outros socorristas de primeira linha, tendo em vista que esses profissionais são cada vez mais chamados em situações que envolvem casos de tentativas de suicídio ${ }^{7}$. Os bombeiros têm exercido um papel significativo no atendimento às vítimas de tentativas de suicídio, sobretudo em relação ao tempo-resposta no atendimento e nas intervenções precoces ${ }^{8}$.

Desse modo, reconhece-se a importância da capacitação das equipes de bombeiros para lidarem com as peculiaridades do comportamento suicida, garantindo abordagem e encaminhamento adequados às vítimas de tentativas de suicídio ${ }^{6}$, visto que dar especial atenção à pessoa que tentou suicidar-se é uma das principais estratégias para evitar um futuro suicídio9,10. Assim, a realização de estudos a partir de dados do Corpo de Bombeiros pode contribuir para fundamentar a assistência promovida por esses profissionais.

Nessa perspectiva, este estudo tem como objetivo descrever o perfil das vítimas de tentativas de suicídio atendidas pelo Corpo de Bombeiros de um município do semiárido brasileiro, no período entre 2000 e 2017.

\section{MÉTODOS}

Trata-se de um estudo epidemiológico, de corte transversal, que utilizou dados da fase pré-hospitalar de atendimentos a vítimas de tentativas de suicídio, realizado pelo $70 \mathrm{Grupa}-$ mento de Bombeiros Militar do Estado de Alagoas (70 GBM), no período de $1^{\circ}$ de janeiro de 2000 a 31 de dezembro de 2017. Os bombeiros militares são acionados, entre outras demandas, para o resgate de vítimas de tentativas de suicídio, principalmente para aquelas tentativas que envolvem situações de risco, como em casos de precipitação de lugares elevados e de afogamento.

O 70 GBM está localizado em Arapiraca, Alagoas e, além desse município, assiste as populações dos municípios de Coité do Nóia, Taquarana, Limoeiro de Anadia, Campo Alegre, São Miguel dos Campos, Lagoa da Canoa, Girau do Ponciano, Craíbas, Campo Grande, Feira Grande, São Sebastião, Junqueiro, Teotônio Vilela e Traipu, totalizando uma população de 427.938 habitantes ${ }^{11}$.

Os dados foram coletados por meio das fichas de atendimento pré-hospitalar e foram armazenados em uma planilha eletrônica de dados (Microsoft Excel' 2010). Foi utilizado um formulário validado por teste piloto, com as seguintes variáveis: sexo; idade; idade maior ou menor de 18 anos; método utilizado para a tentativa de suicídio; tentativa de suicídio por intoxicação; uso de bebida alcoólica; dia da semana em que ocorreu a tentativa de suicídio; horário da ocorrência; mês da ocorrência; ano da ocorrência; estação do ano; presença de transtorno mental; ocorrência de óbito após o acionamento do Corpo de Bombeiros. A variável denominada "tentativa de suicídio por intoxicação" equivale a todos os casos de intoxicação por medicamentos, venenos e outras substâncias. O horário em que ocorreu a tentativa, bem como a presença de transtorno mental, foi informado pela família durante a ligação de acionamento do $70 \mathrm{GBM}$. Os dados do teste piloto foram incorporados aos resultados dessa pesquisa.

Os intervalos de tempo considerados no atendimento pré-hospitalar (em minutos) foram analisados: tempo de resposta (entre o acionamento para o atendimento do $70 \mathrm{GBM}$ até a chegada à cena do evento), tempo de transporte (entre a saída da cena até a chegada ao hospital) e tempo total (desde o acionamento até a chegada ao hospital de destino, ou finalização da ocorrência) ${ }^{6}$. 
Os dados foram analisados por meio dos programas Epi Info ${ }^{\text {TM }} 7$ e SPSS versão 20.0. Inicialmente, foi realizada análise individual das variáveis, por meio da distribuição de frequências e medidas de tendência central. Os dados referentes às categorias "informação ignorada" ou "informação imprecisa" foram excluídos da descrição. Por esse motivo, existe diferença no total de cada uma das variáveis.

A normalidade dos dados foi avaliada pelo teste de Kolmogorov-Smirnov. Para identificar possíveis associações entre as tentativas de suicídio, segundo sexo e variáveis independentes, foram utilizados o teste do qui-quadrado, o teste exato de Fisher e o teste de Kruskal-Wallis. Na análise, adotou-se significância de 5\%.

Este estudo foi aprovado pelo Comitê de Ética em Pesquisa da Universidade Federal de Alagoas (UFAL), sob o parecer n’ 2.744.463/2018, conforme diretrizes da Resolução no 466/2012 sobre pesquisas envolvendo seres humanos.

\section{RESULTADOS}

Entre os anos de 2000 e 2017, foram realizados 14.589 atendimentos pelo $70 \mathrm{GBM}$, dos quais, 144 foram referentes a vítimas de tentativas de suicídio. Desses, sete já haviam sido consumados quando a equipe chegou para o resgate ( 5 homens e 2 mulheres) e um foi a óbito ao chegar à unidade hospitalar. Esses óbitos foram incluídos no estudo, pois as chamadas referiam-se a "tentativas de suicídio". Na distribuição, ao longo dos 18 anos estudados, os anos de 2004 $(n=28 ; 19,4 \%), 2005(n=22 ; 15,3 \%)$ e $2006(n=22 ; 15,3 \%)$ totalizaram 72 atendimentos, concentrando $50 \%$ dos casos atendidos.

Entre as vítimas atendidas, 73 (50,7\%) eram do sexo masculino e 71 (49,3\%), do sexo feminino. A média geral de idade foi de $30,7( \pm 12,44)$ anos, apresentando os homens a média de 33,41 ( $\pm 12,15)$ anos e as mulheres, de 27,94 $( \pm 12,21)$ anos. Ao comparar as médias de idades entre os sexos das vítimas, foi encontrada diferença estatística ( $K W=8,99 ; \mathrm{p}<0,001$ ), indicando que as mulheres possuíam idade inferior à dos homens.

Ao observar as vítimas menores de 18 anos $(n=14 ; 100 \%)$, verificou-se que todas eram do sexo feminino. O método utilizado para a tentativa entre elas foi a intoxicação $(n=10$; $71,5 \%)$, seguida pela arma branca $(n=3 ; 21,4 \%)$, e em um caso essa informação foi ignorada $(n=1 ; 7,1 \%)$. Na amostra geral, o método mais utilizado pelas vítimas de tentativas de suicídio foi a intoxicação $(n=73 ; 50,7 \%)$. Ao comparar a variável tentativa de suicídio por intoxicação com a variável sexo, observou-se diferença estatística entre homens e mulheres $(p<0,001)$, no qual estas $(n=49 ; 67,12 \%)$ utilizaram a intoxicação em uma maior frequência, quando comparada àqueles $(n=24 ; 32,9 \%)$ (Tabela 1). As intoxicações identificadas foram por medicação ( $n=30 ; 41,09 \%)$, pesticida ( $n=29$; $39,73 \%)$ e água sanitária $(n=5 ; 6,85 \%)$. Em 9 casos (12,33\%) não houve registro da substância utilizada (Tabela 1).

Sobre o uso de álcool, identificou-se que 9,3\% ( $n=11)$ das pessoas atendidas fizeram uso de bebida alcoólica, dos quais $72,72 \%(n=8)$ eram do sexo masculino. Em 18,1\% ( $n=$ 26) dos casos essa informação foi ignorada. Não foi encontrada significância estatística entre o consumo de álcool e a variável sexo, provavelmente em razão da ausência de dados. Destaca-se que dessas fichas sem registro $(n=26)$ sobre a utilização de álcool, não havia diferença entre sexo (14 eram homens e 12 mulheres) e faixa etária (distribuição aleatória) (Tabela 1).

O desfecho letal, apresentado na tabela 2, refere-se aos casos de tentativas de suicídio em que as vítimas foram a óbito após o acionamento do Corpo de Bombeiros. Dos oito óbitos ocorridos, sete $(87,5 \%)$ aconteceram antes de a equipe de bombeiros chegar ao local de resgate e um $(12,5 \%)$ deles, após a equipe chegar ao hospital de referência. Os métodos utilizados para os desfechos letais foram: enforcamento (dois homens), precipitação de lugar elevado (uma mulher), intoxicação (um homem) e afogamento (uma mulher). Em três dos óbitos ocorridos (dois homens e uma mulher), a informação sobre o método utilizado não foi registrada.

Já no que se refere à presença de transtorno mental, observou-se significância estatística entre os sexos ( $p=$ $0,04)$, havendo maior frequência de casos no sexo masculino $(76,5 \% ; n=13)$ do que no sexo feminino $(23,5 \% ; n=4)$ (Tabela 1).

Na tabela 2, observa-se que as tentativas de suicídio ocorreram com maior frequência no verão $(n=40,27,8 \%)$ e na primavera $(n=39,27,1 \%)$. A sexta-feira foi o dia da semana com maior número de atendimentos ( $n=31 ; 21,5 \%$ ), seguida da terça-feira ( $n=24 ; 16,7 \%$ ). $O$ horário predominante para as tentativas de suicídio foi entre 12h e 17h59min ( $\mathrm{n}=$ $60 ; 41,7 \%)$.

Ao comparar os diferentes métodos utilizados para a tentativa de suicídio com a variável sexo, as intoxicações foram predominantes nas mulheres ( $n=49 ; 67,12 \%$ ), enquanto queimaduras $(n=3 ; 100 \%)$, arma de fogo $(n=2 ; 100 \%)$, precipitação de lugar elevado ( $n=12 ; 80 \%)$, enforcamento ( $n=$ $3 ; 75 \%)$ e arma branca ( $n=12 ; 66,7 \%)$ foram mais prevalentes nos homens (Tabela 2).

Quanto à média do tempo consumido, em minutos, na fase do atendimento pré-hospitalar entre homens e mulheres, observou-se diferença estatística no tempo de transporte $(p=0,01)$, que apresentou um consumo maior no atendimento aos homens. Entre os atendimentos cujo tempo total consumido foi maior que uma hora, $90 \%(n=9)$ foram de homens, e em 70\% ( $n=7)$ desses casos o método utilizado foi a precipitação de lugar elevado (Tabela 3). 
Tabela 1. Características das tentativas de suicídio atendidas pelo $7^{\circ}$ GBM segundo sexo, entre os anos de 2000 e 2017, em Arapiraca, Alagoas, Brasil

\begin{tabular}{|c|c|c|c|c|c|c|c|c|c|}
\hline \multirow{3}{*}{ Característica } & \multicolumn{4}{|c|}{ Sexo } & \multirow{2}{*}{\multicolumn{2}{|c|}{ Total ${ }^{\star \star}$}} & \multirow{3}{*}{ OR (IC-95\%) } & \multirow{3}{*}{$x^{2}$} & \multirow{3}{*}{$\mathbf{p}$} \\
\hline & \multicolumn{2}{|c|}{ Masculino } & \multicolumn{2}{|c|}{ Feminino } & & & & & \\
\hline & $\mathbf{N}$ & $\%$ & $\mathbf{N}$ & $\%$ & $N$ & $\%$ & & & \\
\hline Faixa etária & & & & & & & Indefinido & 0,00 & $<0,001^{\star}$ \\
\hline$<18$ & 0 & 0 & 14 & 100 & 14 & 100 & & & \\
\hline$\geq 18$ & 71 & 55,91 & 56 & 44,09 & 127 & 100 & & & \\
\hline Intoxicação & & & & & & & $0,17(0,08-0,39)$ & 19,01 & $<0,001$ \\
\hline Sim & 24 & 32,88 & 49 & 67,12 & 73 & 100 & & & \\
\hline Não & 34 & 73,91 & 12 & 26,09 & 46 & 100 & & & \\
\hline Fez uso de álcool & & & & & & & $2,62(0,66-10,40)$ & 0,14 & 0,21 \\
\hline $\operatorname{Sim}$ & 8 & 72,73 & 3 & 27,27 & 11 & 100 & & & \\
\hline Não & 54 & 50,47 & 53 & 49,53 & 107 & 100 & & & \\
\hline Desfecho & & & & & & & $1,67(0,38-7,25)$ & 0,37 & 0,72 \\
\hline Letal & 5 & 62,5 & 3 & 37,5 & 8 & 100 & & & \\
\hline Não letal & 68 & 50,0 & 68 & 50,0 & 136 & 100 & & & \\
\hline Transtorno mental & & & & & & & $3,38(1,03-11,04)$ & 0,03 & $0,04^{*}$ \\
\hline $\operatorname{Sim}$ & 13 & 76,47 & 4 & 23,53 & 17 & 100 & & & \\
\hline Não & 51 & 49,04 & 53 & 50,96 & 104 & 100 & & & \\
\hline
\end{tabular}

* Teste de Fisher.

${ }^{\star *}$ A soma do total em cada variável varia devido à exclusão de casos para os quais não havia informação ou a informação foi imprecisa.

Tabela 2. Características das tentativas de suicídio atendidas pelo $7^{\circ} \mathrm{GBM}$, entre os anos de 2000 e 2017, em Arapiraca, Alagoas, Brasil

\begin{tabular}{|c|c|c|c|c|c|c|c|c|}
\hline \multirow{3}{*}{ Característica } & \multicolumn{4}{|c|}{ Sexo } & \multirow{2}{*}{\multicolumn{2}{|c|}{ Total ${ }^{\star \star}$}} & \multirow{3}{*}{$\mathrm{X}^{2}$} & \multirow{3}{*}{ p } \\
\hline & \multicolumn{2}{|c|}{ Masculino } & \multicolumn{2}{|c|}{ Feminino } & & & & \\
\hline & $\mathbf{N}$ & $\%$ & $\mathbf{N}$ & $\%$ & $\mathbf{N}$ & $\%$ & & \\
\hline Estação do ano & & & & & & & 2,33 & 0,51 \\
\hline Verão & 19 & 47,50 & 21 & 52,50 & 40 & 27,78 & & \\
\hline Outono & 16 & 48,48 & 17 & 51,52 & 33 & 22,92 & & \\
\hline Inverno & 20 & 62,50 & 12 & 37,50 & 32 & 22,22 & & \\
\hline Primavera & 18 & 46,15 & 21 & 53,85 & 39 & 27,08 & & \\
\hline Dia da semana & & & & & & & 6,57 & 0,36 \\
\hline Domingo & 14 & 63,64 & 8 & 36,36 & 22 & 15,28 & & \\
\hline Segunda & 11 & 64,71 & 6 & 35,29 & 17 & 11,81 & & \\
\hline Terça & 11 & 45,83 & 13 & 54,17 & 24 & 16,67 & & \\
\hline Quarta & 7 & 36,84 & 12 & 63,16 & 19 & 13,19 & & \\
\hline Quinta & 8 & 47,06 & 9 & 52,94 & 17 & 11,81 & & \\
\hline Sexta & 13 & 41,94 & 18 & 58,06 & 31 & 21,53 & & \\
\hline Sábado & 9 & 64,29 & 5 & 35,71 & 14 & 9,72 & & \\
\hline Faixa horária & & & & & & & 7,29 & $0,06^{*}$ \\
\hline $6 \mathrm{~h}$ às 11h59min & 15 & 53,57 & 13 & 46,43 & 28 & 19,44 & & \\
\hline $12 \mathrm{~h}$ às $17 \mathrm{~h} 59 \mathrm{~min}$ & 33 & 55,00 & 27 & 45,00 & 60 & 41,67 & & \\
\hline $18 \mathrm{~h}$ às 23h59min & 12 & 33,33 & 24 & 66,67 & 36 & 25,00 & & \\
\hline 0h às 5h59min & 10 & 71,43 & 4 & 28,57 & 14 & 9,72 & & \\
\hline Natureza da ocorrência & & & & & & & 21,9 & $<0,001^{*}$ \\
\hline Afogamento & 2 & 50,00 & 2 & 50,00 & 4 & 2,78 & & \\
\hline Arma branca & 12 & 66,67 & 6 & 33,33 & 18 & 12,50 & & \\
\hline Arma de fogo & 2 & 100,00 & 0 & 0,00 & 2 & 1,39 & & \\
\hline Enforcamento & 3 & 75,00 & 1 & 25,00 & 4 & 2,78 & & \\
\hline Intoxicação & 24 & 32,88 & 49 & 67,12 & 73 & 50,69 & & \\
\hline Precipitação & 12 & 80,00 & 3 & 20,00 & 15 & 10,42 & & \\
\hline Queimaduras & 3 & 100,00 & 0 & 0,00 & 3 & 2,08 & & \\
\hline
\end{tabular}

* Teste de Fisher.

** A soma do total em cada variável varia devido à exclusão de casos para os quais não havia informação ou a informação foi imprecisa. 
Tabela 3. Distribuição da média de tempo consumido (em minutos), em cada fase de atendimento às vítimas de tentativas de suicídio, segundo o sexo, entre os anos de 2000 e 2017, em Arapiraca, Alagoas, Brasil

\begin{tabular}{|c|c|c|c|c|}
\hline \multirow{3}{*}{ Tempos consumidos } & \multicolumn{2}{|c|}{ Sexo } & \multirow{3}{*}{ Teste - H } & \multirow{3}{*}{ p } \\
\hline & Masculino & Feminino & & \\
\hline & Média & Média & & \\
\hline Tempo de resposta & 9,54 & 10,02 & 0,46 & 0,50 \\
\hline Tempo de transporte & 35,42 & 15,71 & 6,12 & 0,01 \\
\hline Tempo total & 44,36 & 25,94 & 2,42 & 0,12 \\
\hline
\end{tabular}

Após o atendimento realizado pelo Corpo de Bombeiros, a maior parte das vítimas foi encaminhada para o hospital de emergência de referência para tentativas de suicídio ( $n=109,75,6 \%)$; quatro $(2,8 \%)$ ficaram no local sob a responsabilidade da equipe do Serviço de Atendimento Móvel de Urgência (SAMU), para assistência das lesões; quatro $(2,8 \%)$ foram encaminhadas para o Centro de Atenção Psicossocial (CAPS); uma $(0,7 \%)$ foi encaminhada para um hospital psiquiátrico; em 19 (13,2\%) fichas de atendimentos essa informação estava ignorada. Os sete casos $(4,9 \%)$ em que as vítimas foram a óbito antes de a equipe chegar ao resgate, ficaram sob os cuidados da Polícia Civil ou Militar e do Instituto Médico Legal (IML).

\section{DISCUSSÃO}

O atendimento pré-hospitalar é considerado o primeiro serviço a entrar em contato com a vítima, que necessita de cuidados imediatos e cuja assistência pode influenciar diretamente no prognóstico do paciente ${ }^{12-14}$. Nas situações que envolvem emergências, tais como as tentativas de suicídio, os primeiros mediadores desse tipo de situação são, muitas vezes, bombeiros, que trabalham em situações de crise em que são necessárias intervenções imediatas e eficientes ${ }^{7}$.

Nessa perspectiva, a OMS ${ }^{15,16}$ lançou, em 2004, algumas recomendações para a prevenção do comportamento suici$\mathrm{da}$, das quais uma delas foi promover treinamento para profissionais que se encontram na linha de frente e que primeiro entram em contato com pessoas sob risco de suicídio.

No manual intitulado "Prevenção do suicídio: um recurso para policiais, bombeiros e outros socorristas"7, de 2009, pesquisadores da OMS recomendam que esses profissionais sejam treinados para: detecção precoce dos fatores de risco para as tentativas de suicídio; reconhecimento dos sinais e sintomas de transtorno mental; controle do acesso aos meios letais; compreensão do funcionamento da rede de serviços de saúde mental disponíveis localmente e como acessá-los, entre outras recomendações.

No que diz respeito ao perfil de tentativas de suicídio segundo o sexo, verificou-se que a frequência de tentativas cometidas por homens e mulheres foi semelhante, o que difere de estudos nacionais e internacionais, que apontam para a predominância de tentativas de suicídio entre as mulheres ${ }^{6,8,13,17}$. Possivelmente, o perfil encontrado está relacionado ao fato de que o Corpo de Bombeiros é o serviço pré-hospitalar acionado para os casos de emergência que envolvem situações mais arriscadas, tanto para os profissionais que realizam o atendimento como para as vítimas a serem assistidas. Desse modo, a própria dinâmica do serviço pode estar relacionada ao perfil de vítimas identificado neste estudo, visto que os homens são os que utilizam predominantemente métodos mais letais, como arma de fogo, arma branca e precipitação de lugares elevados,6,13,17.

Em relação à idade das vítimas, observa-se que 10\% dos casos de tentativas de suicídio ocorreram entre adolescentes. Estima-se que, nessa fase da vida, de 3\% a 20\% das pessoas poderão tentar suicídio ${ }^{18}$, visto que a adolescência pode ser considerada como uma fase estressante, devido às inúmeras e intensas mudanças físicas e psicológicas ocorridas nesse período ${ }^{19}$. Desse modo, o apoio psicológico e familiar é essencial para que o jovem descubra suas aptidões e suas oportunidades ${ }^{20}$ e sinta-se acolhido e capaz, pois muitos podem tentar o suicídio como uma forma de pedir ajuda, uma vez que comumente estão vivenciando algum tipo de conflito ou transtorno mental21.

Quanto ao método utilizado para as tentativas de suicídio, verificou-se que a intoxicação foi o mais utilizado em ambos os sexos, principalmente pelas mulheres, que optaram duas vezes mais por esse método do que os homens. Esses achados estão em conformidade com os resultados obtidos na literatura ${ }^{6,13,22}$, que apontam que as mulheres recorrem aos métodos mais brandos. Em estudo transversal ${ }^{23}$, com casos de tentativas de suicídio por intoxicações exógenas em um município do Mato Grosso, dos 164 casos analisados, $70,1 \%$ eram mulheres e $29,1 \%$, homens. Os agentes tóxicos utilizados com maior frequência foram os medicamentos, principalmente os que apresentavam ação no sistema nervoso e os agrotóxicos, com destaque para os rodenticidas e os piretroides ${ }^{23}$.

O uso de álcool esteve presente em 9,3\% dos atendimentos por tentativas de suicídio. Entretanto, em 18,1\% dos atendimentos, não houve registro referente a essa informação. A literatura apresenta associação entre o uso abusivo dessa substância e as tentativas de suicídio, principalmente entre os homens ${ }^{6,17,21}$. Em estudo transversal ${ }^{24}$ realizado com 632 laudos de vítimas de suicídio no estado de São Paulo, observou-se que cerca de um terço dos suicídios foi cometido 
após o consumo de álcool. Nesse mesmo estudo $24,37,1 \%$ dos homens e 20,1\% das mulheres tiveram alcoolemia positiva. Acredita-se que o uso de álcool tenha sido maior do que o constatado nesse estudo e que a não associação encontrada entre essa variável e as tentativas de suicídio pode estar relacionada à falta de registro dessa informação. Por isso, faz-se necessária a conscientização dos profissionais quanto ao registro do uso de álcool e de outras informações para o planejamento de estratégias de cuidado e de prevençãó.

No presente estudo, o enforcamento, seguido da precipitação de lugar elevado, intoxicação exógena e afogamento, foi o método utilizado para cometer o suicídio. Segundo a OMS', a ingestão de pesticidas, os enforcamentos e as armas de fogo estão entre os métodos mais comuns de suicídio no mundo. Entretanto, a escolha do método pode ser influenciada pela cultura e pelo acesso que se tem a ele ${ }^{10}$. No Brasil, o método mais utilizado é o enforcamento, seguido da morte por projétil de arma de fogo². Entre 2011 e 2017, o enforcamento foi o meio mais utilizado por brasileiros de 15 a 29 anos de idade ${ }^{3}$, principalmente no sexo masculino; seguido pela intoxicação exógena, que foi mais frequente no sexo feminino; e pela arma de fogo, que foi mais utilizada pelos homens do que pelas mulheres. No Japão ${ }^{25}$, em um estudo baseado em 873.268 casos de suicídios, ocorridos entre 1974 e 2014, o método mais utilizado foi o enforcamento, seguido pela precipitação de altura e por exposição a gás.

Como estratégia para a prevenção do suicídio, a OMS recomenda a estruturação de políticas públicas que restrinjam o acesso aos meios utilizados, como a limitação de acesso a pesticidas e armas de fogos e a construção de barreiras nas pontes. Em uma revisão sistemática ${ }^{26}$, realizada com o objetivo de investigar os efeitos de restrições de vendas de pesticidas sobre a incidência de suicídios em diferentes países, os resultados demonstraram que essas ações foram eficazes na redução das taxas de suicídio.

A presença de transtorno mental, registrada segundo relato de familiares, foi predominante no sexo masculino (76,5\%). Esse resultado pode estar relacionado ao fato de que a população masculina procura menos os serviços de saúde do que as mulheres. Sem acompanhamento da doença mental, os homens possuem maior risco de tentar o suicídio. Já na população feminina, o reconhecimento precoce dos sinais de risco de depressão, suicídio e doença mental reduz essas chances ${ }^{8}$. Em um estudo realizado por Rutz et al. ${ }^{27}$ sobre a prevenção de suicídio, em Gotland, Suécia, os autores destacaram que até $70 \%$ dos suicídios ocorreram durante a presença de transtorno depressivo e que os homens com depressão eram incapazes de pedir ajuda. Nesse mesmo estudo, as pessoas que apresentaram depressão foram tratadas na atenção primária, ou encaminhadas a uma clínica psiquiátrica local, levando à diminuição significativa da frequência de suicídios e de internamentos para o tratamento da depressão. Esse é um bom exemplo de como uma intervenção que inclui a capacitação profissional visando ao tratamento adequado da depressão pode reduzir o número de suicídios ${ }^{27}$.

No presente estudo, as tentativas de suicídio ocorreram com maior frequência no verão e na primavera. No nordeste brasileiro, o clima da região é definido por períodos de seca e de chuva. Assim, é provável que certas atividades sociais e laborais, a exemplo do trabalho dos agricultores, tenham uma relação com as variações climáticas, podendo exercer influência sobre o comportamento suicida ${ }^{28-30}$. Os resultados de um estudo realizado na província de Shandong, China ${ }^{29}$, com o objetivo de examinar a sazonalidade do suicídio, evidenciaram que as variações sazonais do suicídio foram mais acentuadas nas áreas rurais, em particular pela ingestão de pesticidas ${ }^{29}$.

Quanto aos dias da semana, verificou-se que as tentativas de suicídio aconteceram com maior frequência nos dias de sexta-feira e terça-feira, divergindo de estudos anteriores ${ }^{6,29}$. Em estudo realizado com 2.142 vítimas de tentativas de suicídio, atendidas em um hospital de emergência do nordeste brasileiro, os dias da semana mais frequentes para as tentativas foram sábado, domingo e segunda-feira ${ }^{31}$. Possivelmente, as maiores frequências de tentativas de suicídio durante as sextas e terças-feiras podem ter ocorrido devido ao fato de que, durante os finais de semana, as pessoas podem não ter sido capazes de tentar o suicídio por estarem acompanhadas de familiares e amigos ${ }^{25}$.

No presente estudo, observou-se que as tentativas de suicídio foram mais frequentes durante os períodos da tarde e da noite, o que se assemelha a estudos anteriores ${ }^{6,30}$. Entretanto, os fatores que podem ter contribuído para a maior frequência de tentativas nessas faixas horárias são desconhecidos ${ }^{30}$.

Em relação ao atendimento pré-hospitalar, o tempo consumido, nessa fase da assistência, é crucial para a manutenção da vida e resulta de um conjunto de fatores que o influenciam$^{6}$. Neste estudo, constatou-se que a média do tempo de transporte consumido no atendimento aos homens foi significativamente maior, quando comparado com o tempo de transporte consumido no atendimento às mulheres, o que se assemelha a um estudo realizado anteriormente em Arapiraca, Alagoas, Brasil' ${ }^{6}$. Tal diferença pode estar relacionada ao fato de os homens utilizarem métodos mais letais, que demandam maiores cuidados, pois, entre os atendimentos cujo tempo total foi acima de uma hora (6,9\%), 90\% foram de homens e, em 70\% desses casos, o meio utilizado foi a precipitação de lugar elevado.

Este estudo deve ser analisado levando-se em consideração algumas limitações, pois a utilização das fichas de ocorrência, como fonte de informação, apresentam desvantagens e limitações metodológicas, como o viés de informação, preenchimento incompleto, dificuldades de obtenção de dados decorrentes do tipo de exposição e ocultação 
das informações colhidas da própria vítima e de terceiros. Somado a esses fatores, há ainda a dificuldade em avaliar se o episódio foi acidental ou se houve uma intenção suicida ${ }^{8,14}$.

\section{CONCLUSÃO}

Ao descrever o perfil das vítimas de tentativas de suicídio atendidas pelo Corpo de Bombeiros de um município do semiárido brasileiro, observa-se diferenças entre homens e mulheres, com destaque para os seguintes achados: a frequência de atendimentos entre homens e mulheres foi semelhante, mas com diferença estatística entre as suas idades. A intoxicação foi o método mais comum entre as vítimas, e as mulheres fizeram uso desse método duas vezes mais do que os homens. Observou-se que a presença de transtorno mental foi mais frequente entre os homens, e estes foram os que mais demandaram tempo de atendimento pelo serviço pré-hospitalar, indicando uma possível maior gravidade nos casos de tentativas de suicídio.

Esses dados são relevantes para a formulação de estratégias de prevenção das tentativas de suicídio, pois percebe-se que a referida instituição tem um papel importante no atendimento a essas vítimas, principalmente entre aquelas que utilizaram como método a precipitação de lugar elevado. Os bombeiros devem entender esse momento como uma complexa experiência existencial, favorecendo o diálogo e adotando uma abordagem acolhedora na atenção a essas vítimas. Fazem-se necessários, ainda, o reconhecimento precoce dos casos de tentativas de suicídio, as principais formas de atuação e o preenchimento adequado das fichas de atendimento pré-hospitalar.

\section{CONTRIBUIÇÕES INDIVIDUAIS}

Jefferson Wladimir Tenório de Oliveira - Contribuiu na concepção e elaboração do estudo, análise e interpretação dos dados, elaboração do artigo e aprovação da versão final a ser publicada.

Ana Paula Nogueira de Magalhães - Contribuiu na concepção e elaboração do estudo, análise e interpretação dos dados, elaboração do artigo e revisão do conteúdo, e aprovação da versão final a ser publicada.

Elaine Kristhine Rocha Monteiro - Contribuiu na análise e interpretação dos dados, elaboração do artigo e aprovação da versão final a ser publicada.

Alice Correia Barros - Contribuiu na análise e interpretação dos dados, elaboração do artigo e aprovação da versão final a ser publicada.

Verônica de Medeiros Alves - Contribuiu na análise e interpretação dos dados, elaboração do artigo e revisão do conteúdo, e aprovação da versão final a ser publicada.
Carlos Dornels Freire de Souza - Contribuiu na análise e interpretação dos dados, elaboração do artigo e revisão do conteúdo, e aprovação da versão final a ser publicada.

\section{CONFLITO DE INTERESSES}

Os autores declaram não haver conflito de interesses nem financeiros a serem declarados.

\section{REFERÊNCIAS}

1. World Health Organization. Preventing suicide: a global imperative [Internet]. Luxemburgo: World Health Organization; 2014 [cited 2019 Sep 12]. Disponível em: https://www.who. int/mental_health/suicide-prevention/world_report_2014/en/. Acesso em: jun. 2019.

2. Santos MSP, Silva TPS, Pires CMC, Ramos PGX, Sougey EB. Identificaçãao de aspectos associados à tentativa de suicídio por envenenamento. J Bras Psiquiatr. 2017;66(4):197-202.

3. Brasil. Ministério da Saúde, Secretaria de Vigilância em Saúde. Boletim Epidemiológico v. 50, n 24: Perfil epidemiológico dos casos notificados de violência autoprovocada e óbitos por suicídio entre jovens de 15 a 29 anos no Brasil, 2011 a 2018 [Internet]. Braślila: Ministério da Saúde; 2019 [cited 2019 Sep 14]. Disponível em: https://portalarquivos2.saude.gov.br/ images/pdf/2019/setembro/13/BE-suic--dio-24-final.pdf. Acesso em: jun. 2019.

4. Nock MK, Borges G, Bromet EJ, Cha CB, Kessler RC, Lee S. Suicide and Suicidal Behavior. Epidemiol Rev. 2008;30(1):133-54.

5. Bertolote JM, Mello-Santos C, Botega NJ. Detecting suicide risk at psychiatric emergency services. Rev Bras Psiquiatr. 2010;32(2):87-95.

6. Magalhães APN, Alves VM, Comassetto I, Lima PC, Faro ACM, Nardi AE. Atendimento a tentativas de suicídio por serviço de atenção pré-hospitalar. J Bras Psiquiatr. 2014;63(1):16-22.

7. World Health Organization. Department of Mental Health. Preventing Suicide: a resource for police, firefighters and other first line responders [Internet]. Genebra: World Health Organization; 2009 [cited 2019 Sep 1]. Available from: https://www.who.int/mental_ health/prevention/suicide/resource_firstresponders.pdf. Acesso em: jun. 2019.

8. Santana JCB, Dutra BS, Souza HNF, Moura IC, Faria RAD, Hang-Costa TA, et al. Caracterização das vítimas de tentativa de autoextermínio atendidas pelo Serviço de Atendimento Móvel de Urgência (SAMU) no município de Sete Lagoas e região. Rev Bioethikos. 2011;5(1):84-92.

9. Souza VS, Alves MS, Silva LA, Lino DCSF, Nery AA, Casotti CA. Tentativas de suicídio e mortalidade por suicídio em um município no interior da Bahia. J Bras Psiquiatr. 2011;60(4):4-300.

10. Botega NJ. Comportamento suicida: epidemiologia. Psicologia USP. 2014;25(3):231-6.

11. Instituto Brasileiro de Geografia e Estatística (IBGE) [Internet]. Rio de Janeiro: IBGE; 2019 [cited 2019 mar 6]. Disponível em: http://www.ibge.gov.br/cidadesat/topwindow.htm?1. Acesso em: jun. 2019.

12. Abasse MLF, Oliveira RC, Silva TC, Souza ER. Análise epidemiológica da morbimortalidade por suicídio entre adolescentes em Minas Gerais, Brasil. Ciênc Saúde Colet. 2009;14(2):407-16.

13. Alves VM, Silva AMS, Magalhães APN, Andrade TG, Faro ACM, Nardi AE. Suicide attempts in a emergency hospital. Arq Neuropsiquiatr. 2014;72(2):123-8.

14. Rosa NM, Dell Agnolo CM, Oliveira RR, Mathias TAF, Oliveira MLF. Tentativas de suicídio e suicídios na atenção pré-hospitalar. J Bras Psiquiatr. 2016;65(3):231-8.

15. World Health Organization. For which strategies of suicide prevention is there evidence of effectiveness. Regional Office for Europe's Health Evidence Network (HEN). Geneva: WHO; 2004.

16. Botega NJ, Werlang BSG, Cais CFS, Macedo MMK. Prevenção do comportamento suicida. Psico. 2006;37(3):213-20.

17. Cavalcante FG, Minayo MCS. Estudo qualitativo sobre tentativas e ideações suicidas com 60 pessoas idosas brasileiras. Ciênc Saúde Colet. 2015;20(6):1655-66.

18. Vidal CEL, Gontijo ECDM, Lima LA. Tentativas de suicídio: fatores prognósticos e estimativa do excesso de mortalidade. Cad Saúde Pública. 2013;29(1):175-87. 
19. Claumann GS, Pinto AA, Silva DAS, Pelegrini A. Prevalência de pensamentos e comportamentos suicidas e associação com a insatisfação corporal em adolescentes. J Bras Psiquiatr. 2018;67(1):3-9.

20. Pinto LLT, Meira SS, Ribeiro IJS, Nery AA, Casotti CA. Tendência de mortalidade por lesões autoprovocadas intencionalmente no Brasil no período de 2004 a 2014. J Bras Psiquiatr. 2017;66(4):203-10

21. Hildebrandt LM, Zart F, Leite MT. A tentativa de suicídio na percepção de adolescentes: um estudo descritivo. Rev Eletr Enf. 2011;13(2):219-26.

22. Ficher AMFT, Varsan GA. Tentativas de suicídio em jovens: aspectos epidemiológicos dos casos atendidos no setor de urgências psiquiátricas de um hospital geral universitário entre 1988 e 2004. Estud Psicol. 2008;25(3):361-74.

23. Vieira LP, Santana VTP, Suchara EA. Caracterização de tentativas de suicídios por substâncias exógenas. Cad Saúde Colet. 2015;23(2):118-23.

24. Ponce JC, Andreuccetti G, Jesus MGS, Leyton V, Muñoz DR. Álcool em vítimas de suicídio em São Paulo. Rev Psiquiatr Clín. 2008:35(1):13-6.
25. Boo J, Matsubayashib T, Uedac M. Diurnal variation in suicide timing by age and gender: evidence from Japan across 41 years. J Affect Disord. 2019;243:366-74.

26. Gunnell D, Knipe D, Chang S, Pearson M, Konradsen F, Lee WJ, et al. Prevention of suicide with regulations aimed at restricting access to highly hazardous pesticides: a systematic review of the international evidence. Lancet Glob Health. 2017;5(10):1026-37.

27. Rutz W, Knorring LV, Pihlgren H, Rihmer Z, Wålinder J. Prevention of male suicides: lessons from Gotland study. Lancet. 1995;345:524.

28. Coimbra DG, Silva ACP, Sousa-Rodrigues CF, Barbosa FT, Figueredo DS, Santos JLA, et al. Do suicide attempts occur more frequently in the spring too? A systematic review and rhythmic analysis. J Affect Disord. 2016;196:125-37.

29. Sun J, Guo X, Ma J, Zhang J, Jia C, Xu A. Seasonality of suicide in Shandong China, 1991-2009: associations with gender, age, area and methods of suicide. J Affect Disord. 2011;135(1-3):258-66.

30. Alves VM, Francisco LC, Melo AR, Novaes CR, Belo FM, Nardi AE. Trends in suicide attempts at an emergency department. Rev Bras Psiquiatr. 2017;39(1):55-61. 